\title{
Air-gap convection in rotating electrical machines
}

\author{
David A. Howey Member, IEEE, Peter R.N. Childs and Andrew S. Holmes Member, IEEE
}

\begin{abstract}
This paper reviews convective heat transfer within the air-gap of both cylindrical and disc geometry rotating electrical machines, including worked examples relevant to fractional horsepower electrical machines. Thermal analysis of electrical machines is important because torque density is limited by maximum temperature. Knowledge of surface convective heat transfer coefficients is necessary for accurate thermal modelling for example using lumped parameter models. There exists a wide body of relevant literature, but much of it has traditionally been in other application areas, dominated by mechanical engineers, such as gas turbine design. Particular attention is therefore given to the explanation of the relevant non-dimensional parameters, and to the presentation of measured convective heat transfer correlations for a wide variety of situations from laminar to turbulent flow at small and large gap sizes for both radial flux and axial flux electrical machines.
\end{abstract}

Index Terms-Convection, electrical machine, heat transfer, thermal analysis

\section{INTRODUCTION}

$\mathbf{T}$ HE electromagnetic analysis of motors and generators is a mature subject; in contrast, the thermal and aerodynamic aspects of electrical machine design have been less thoroughly researched to date [1]. Modern machines must be compact, light and torque dense and are often required to withstand extreme environmental and loading conditions. Knowledge of the air flow in a machine is crucial for design purposes, particularly where air-gap convection limits heat transfer. The surface convective heat transfer coefficients, important for the calculation of temperatures, are complex functions of geometry and fluid mechanics. This review paper seeks to give an overview of the state of the art in air flow and convection within the air-gap in cylindrical and disc type machines, with a particular emphasis on experimentally measured convection data in the form of correlations that can be applied during design. It also seeks to summarise the typical flow patterns that might arise, some of which are quite counter-intuitive. Other related thermal topics such as lumped parameter modelling, radiation heat transfer, thermal contact resistances and finite element analysis are not discussed in detail since they have been covered elsewhere, e.g. [2], [3].

In an electrical machine the internal temperatures reached at a given operating point must be predicted during design because:

Manuscript received May 31, 2010. Revised version submitted Sep 18 2010. Accepted for publication November 29, 2010.

Copyright (c) 2010 IEEE. Personal use of this material is permitted. However, permission to use this material for any other purposes must be obtained from the IEEE by sending a request to pubs-permissions@ieee.org

D.A. Howey is now based at the Department of Engineering Science, Oxford University, UK (email: david.howey@eng.ox.ac.uk).

P.R.N. Childs is with the Department of Mechanical Engineering, Imperial College, Exhibition Road, London, SW7 2AZ, UK.

A.S. Holmes is with the Department of Electrical and Electronic Engineering, Imperial College, Exhibition Road, London, SW7 2AZ, UK
1) Calculation of the maximum continuous rating requires an adequate thermal model to ensure that the cooling provision is sufficient to avoid overheating, since the torque density (and therefore maximum RMS current density in the windings) is thermally limited in a machine of good electromagnetic design.

2) Stator temperature affects efficiency, because the resistivity of the copper windings increases with temperature. For example, at $100^{\circ} \mathrm{C}$, the resistivity of copper is increased by almost $30 \%$ compared to room temperature.

3) Materials such as permanent magnets and polymers used in stator construction and can only typically withstand continuous maximum temperatures of about $150^{\circ} \mathrm{C}$.

4) Inaccurate predictions lead to the use of excessive safety margins. Improvements would reduce the number of prototype iterations required and minimise cost. This is becoming increasingly important even in smaller machines.

Temperatures that are consistently too high will reduce the lifetime of the machine and may lead to serious failure. The main failure mechanism is breakdown of the insulation surrounding the copper windings due to oxidation and embrittlement. This leads to a loss of mechanical strength and eventually breakage which may cause short circuits [4]. Although electrical machines may usually be overloaded for short periods of time, if the temperature exceeds the maximum acceptable temperature for the insulation class then the lifetime will be shortened, perhaps severely.

A second failure mechanism in permanent magnet (PM) machines is demagnetization of the magnets. The most commonly used PM material is neodymium-iron-boron $(\mathrm{NdFeB})$. The demagnetisation curve of $\mathrm{NdFeB}$ depends strongly on temperature [5]: as magnet temperature increases, the material's crystal structure changes and the magnitudes of $B_{r}$ and $H_{c}$ decrease. Additionally, the 'knee' in the demagnetisation curve moves up; if the magnets are operated below the knee, irreversible change takes place, making it important to ensure magnet temperatures remain modest at all times.

Internal heating in electrical machines is the result of losses. The temperature reached is governed by the balance between heat generated, heat removed and thermal capacity. Major sources of loss are Joule $\left(I^{2} R\right)$ losses in the windings, core losses due to eddy currents and hysteresis, and mechanical losses. Table I shows approximately how these depend on various operating parameters in an electrical machine: $I$ is the stator current and $R$ the stator resistance, $\Omega$ the shaft speed, $m$ the machine mass, $D$ the rotor diameter, $L$ the rotor axial length, $B$ the average magnetic flux density and $\rho$ the air gap fluid density. 
TABLE I

DEPENDENCY OF POWER LOSSES ON MACHINE AND OPERATING PARAMETERS [6], [7]

\begin{tabular}{ll}
\hline \hline Joule loss & $\alpha I^{2} R_{\text {stator }}$ \\
Eddy current loss & $\alpha \Omega^{2} B^{2}$ \\
Hysteresis loss & $\alpha \quad \Omega B^{n}$ \\
Bearing loss & $\alpha \Omega m$ \\
Windage loss & $\alpha \Omega^{3} D^{5} \rho$ (disc machine, $\left.D>>L\right)$ \\
& $\alpha \Omega^{3} D^{4} L \rho$ (drum machine, $\left.L>>D\right)$ \\
\hline
\end{tabular}

A number of factors determine the rate of heat removal in an air-cooled electrical machine. The geometry and materials, surface areas and machine size have an impact. Since losses are roughly proportional to volume $L^{3}$ (where $L$ is a length dimension), but the surface area is proportional to $L^{2}$, cooling may be more of a challenge in larger machines. Additionally, if a machine is totally enclosed, air will recirculate internally and the heat transfer is typically limited by internal heat transfer (for example conduction through insulating materials) and convection around the outside surfaces. However, the heat removal rate may be substantially increased if the machine can be throughflow ventilated, i.e. air continually enters from ambient, passes through and is then expelled.

An aspect of thermal modelling not discussed in detail in this review paper is solid domain thermal modelling. Heat transfer in the solid domain (e.g. in iron, copper, polymers etc.) is by thermal conduction, governed by the heat equation:

$$
\frac{\partial T}{\partial t}=\alpha \nabla^{2} T
$$

where $T$ is the temperature field, $t$ is time and $\alpha$ is the thermal diffusivity of the material. This equation can be solved analytically by integration in 1D cases and by Fourier series expansion in 2D cases. For complex 2D and 3D geometries with many different material types and complicated boundary conditions, lumped parameter methods and numerical methods are usually applied. Lumped parameter (LP) thermal network models are a common way to evaluate the thermal performance of electrical machines. The method assumes that the spatial distribution of temperature within each different material or sub-component is uniform. Where this is not the case, subcomponents can be further broken down into smaller sections to increase the spatial resolution of the model; this is typically done for example within stator windings. The LP method is fast to compute and therefore can handle transient calculations with complex load cycles. It has been widely applied, for example, Mellor et al. [8] developed LP models for totally enclosed fan cooled (TEFC) induction machines; transient results are compared to experiment and shown to be quite accurate. LP methods can equally be applied to disc-type machines, for example Spooner and Chalmers [9] developed an LP model for an axial flux permanent magnet (AFPM) machine and obtained thermal conduction and contact resistances by conducting tests on a dummy rotor. LP models must be carefully 'tuned' with empirical data, such as convective heat transfer correlations, to ensure adequate performance.

In summary, air-gap convection is crucial in thermal modelling of electrical machines in order to achieve accurate results. In certain situations the air-gap convection is the limiting factor for heat transfer, for example in a through-flow ventilated disc machine where all heat is removed by air passing over the stator surface, or in a cylindrical induction machine where there may be significant heating within the rotor.

\section{PREVIOUS REVIEWS}

There are relatively few review papers on the subject of airflow and convection in rotating electrical machines. Boglietti, Cavagnino, Staton et al. [2] reviewed thermal analysis of electrical machines with a particular focus on lumped parameter models. The same authors [3] also reviewed the more challenging areas in thermal analysis, which include contact resistances, stator winding conductivity, and convection coefficients in particular around end windings and where there are complex flowpaths. Staton and Cavagnino [10] reviewed convection in electrical machines, focusing on correlations for natural convection around the external casings of cylindrical geometry machines and also on correlatons for forced cooling with fans or water jackets. They included a small section on air-gap heat transfer. Disc type machine geometries were not considered.

A recent overview of flow in rotating components is given in a report from the Engineering Sciences Data Unit (ESDU) [7]; this addresses discs, cylinders and cavities. Many correlations are given for the calculation of aerodynamic drag in different situations, but the scope of the report does not include heat transfer.

Childs and Long [11] reviewed experimental, numerical and analytical investigations into forced convection heat transfer in annular passages. This is relevant particularly in the design of cylindrical type electrical machines.

\section{NON-DIMENSIONAL GROUPS}

Convection heat transfer data are normally nondimensionalised so that the number of variables in the problem is reduced and results can be applied generally, to a variety of machine sizes.

The most relevant non-dimensional groups here are the Reynolds number $R e$, which encapsulates rotor speed and machine size; the average Nusselt number $N u$ which is the non-dimensional surface convective heat transfer coefficient; $C_{w}$ the non-dimensional air mass flow rate through a machine and geometric parameters such as the gap ratio $G$.

Reynolds number and Nusselt number can be expressed using different characteristic lengths e.g. the rotational Reynolds number $R e_{\theta}$ and Nusselt number $N u$, using the rotor radius; or the gap Reynolds number $R e_{g}$ and gap Nusselt number $N u_{g}$, using the gap size. For cylindrical annuli the Nusselt number is expressed in terms of the hydraulic diameter $D_{h}=2 g$. These various groups are defined as follows:

$$
R e_{g}=\frac{\Omega g R}{\nu}
$$




$$
\begin{gathered}
R e_{\theta}=\frac{\Omega R^{2}}{\nu} \\
N u=\frac{h R}{k} \text { for disc machines } \\
N u=\frac{h D_{h}}{k} \text { for cylindrical machines } \\
G=\frac{g}{R} \\
C_{w}=\frac{\dot{m}}{\mu R}
\end{gathered}
$$

where $R$ is the rotor radius, $\Omega$ is the speed, $\nu$ is the fluid kinematic viscosity (usually at ambient temperature), $h$ is the convective heat transfer coefficient, $k$ is the fluid conductivity (usually at ambient temperature), $L$ is the length of a cylindrical machine, $g$ is the gap size between rotor and stator, $\dot{m}$ is the air mass flow rate and $\mu$ is the dynamic viscosity. There is another non-dimensional group relevant in cylindrical geometry machines, the Taylor number, which provides an indication of the relative effects of inertial forces and viscosity for an annulus with rotation of one or more of the cylindrical surfaces. The Taylor number based on mean annulus radius $r_{m}=(a+b) / 2$ is defined as:

$$
T a_{m}=\frac{\Omega_{a} r_{m}^{0.5}(b-a)^{1.5}}{\nu}
$$

where $a$ and $b$ are the inner and outer annulus diameters respectively.

\section{A. Application to thermal modelling to electrical machines}

There are various ways in which convective heat transfer coefficients can be applied. The usual approach for example in LP models is to obtain a convective heat transfer 'thermal resistance'. First, the appropriate Nusselt number correlation is selected for the situation, for example $N u=A R e_{\theta}^{B}$. The Nusselt number is calculated from the correlation, and then the convective heat transfer coefficient $h$ is calculated from equation 4 or 5 . The convection thermal resistance is then given by:

$$
R=\frac{1}{h A}
$$

where $A$ is the area over which the heat transfer occurs, being in a cylindrical machine the stator or rotor surface bordering the air-gap annulus, and in a disc machine, the circular stator or rotor surface bordering the air-gap space which is also disc shaped. Note: $R$ is typically not constant (as commonly assumed in LP models) but varies as a function of machine geometry and rotor speed.

\section{CYLINDRICAL GEOMETRY MACHINES}

Cylindrical (or 'drum') geometry electrical machines are the most common type of electrical machine. The air-gap is an annulus formed between two concentric cylinders. The airgap magnetic field lines are orientated in the radial direction, so these are also called radial flux machines. There are many

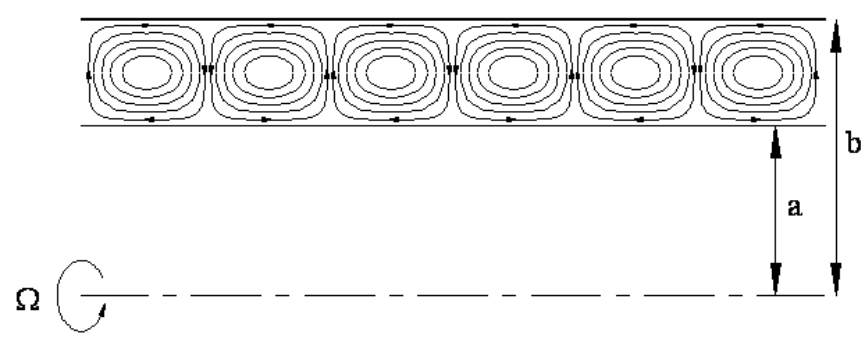

Fig. 1. Taylor vortices in an annulus with rotation

different machine types e.g. induction machines, permanent magnet brushless machines and switched reluctance machines. Flow and heat transfer in this geometry is also important to a wide number of other applications from shafts and axles to spinning projectiles to gas turbine engines.

\section{A. Airflow in the rotor-stator gap}

Couette flow is the term used to describe flow between two surfaces that are in close proximity such that flow is dominated by viscous effects and inertial effects are negligible. In cylindrical coordinates this involves flow in an annulus and the Navier Stokes equations can be solved exactly by analytical techniques, subject to a number of significant assumptions. Couette flow in an annulus with rotation characterises a system in which dynamic equilibrium exists between the radial forces and the radial pressure gradient. However, when it is not possible for the radial pressure gradient and the viscous forces to dampen out and restore changes in centrifugal forces caused by small disturbances in the flow, the fluid motion is unstable and results in a secondary flow. A simple criterion for determining the onset of instability was developed by Rayleigh [12]. In essence the criterion determines whether the force due to inward radial pressure is adequate to maintain inward centripetal acceleration for an arbitrary element of fluid.

In real flows, the onset of this instability is modified by the action of viscosity and rotation of annular surfaces which can lead to instabilities in the flow with the formation of complex toroidal vortices, known for certain flow conditions as Taylor vortices. Taylor vortex flow represents a significant modelling challenge and has been subject to a vast number of studies. Experiments, e.g. Taylor [13], revealed that the simple axisymmetric laminar flow in an annulus with a combination of inner and outer cylinder rotation was replaced by a more complicated eddying flow structure. If the angular velocity exceeded a critical value, a steady axisymmetric secondary flow in the form of regularly spaced vortices in the axial direction were generated as illustrated in figure 1. Alternate vortices rotate in opposite directions.

Taylor [13] formulated the stability problem taking into account the effects of viscosity by assuming an axisymmetric infinitesimal disturbance and solving the dynamic conditions under which instability occurs. Assuming the annular gap size $g=b-a$ is small compared to the mean radius $r_{m}$, known as the small gap approximation, Taylor's solution for the angular 
velocity at which laminar flow breaks down and the flow instabilities grow leading to the formation of secondary flow vortices, called the critical speed, is given by:

$\Omega_{c r}=$

$$
\pi^{2} \nu \sqrt{\frac{a+b}{2 S(b-a)^{3} a^{2}\left[1-\left(\Omega_{b} / \Omega_{a}\right) b^{2} / a^{2}\right]\left(1-\Omega_{b} / \Omega_{a}\right)}}
$$

where:

$$
\begin{aligned}
S= & 0.0571\left[\frac{1+\Omega_{b} / \Omega_{a}}{1-\Omega_{b} / \Omega_{a}}+0.652\left(1-\frac{1}{x}\right)\right]+ \\
& 0.00056\left[\frac{1+\Omega_{b} / \Omega_{a}}{1-\Omega_{b} / \Omega_{a}}+0.652\left(1-\frac{1}{x}\right)\right]^{-1}
\end{aligned}
$$

where $x=a / b$.

For the case of a stationary outer cylinder, $\Omega_{b} / \Omega_{a}=0$ and therefore equations 10 and 11 reduce to:

$$
\begin{gathered}
\Omega_{c r}=\pi^{2} \nu \sqrt{\frac{a+b}{2 S(b-a)^{3} a^{2}}} \\
S=0.0571[1-0.652(b-a) / a]+ \\
0.00056[1-0.652(b-a) / a]^{-1}
\end{gathered}
$$

For the case of a narrow gap and stationary outer cylinder, $x \rightarrow 1$ so $\Omega_{a} / \Omega_{b}=0$ and equations 10 and 11 give a critical speed which results in a critical Taylor number:

$$
T a_{m, c r}=\sqrt{1697}=41.19
$$

If the Taylor number exceeds this critical Taylor number then Taylor vortices can form whereas if $T a_{m}<T a_{m, c r}$ then the flow remains Couette flow.

The critical angular velocity for this case is:

$$
\Omega_{c r}=\frac{41.19 \nu}{r_{m}^{0.5}(b-a)^{1.5}}
$$

Figure 2 shows the variation in Taylor number with rotor size and speed for the specific case of a machine with narrow gap size $0.5 \mathrm{~mm}$. The air viscosity was $\nu=18.9 \mathrm{e}-6 \mathrm{~m}^{2} / \mathrm{s}$, assuming an air temperature of about $60^{\circ} \mathrm{C}$. It can be seen that at higher speeds and larger rotor radii there may be Taylor vortices in the gap since the Taylor number is greater than the critical Taylor number, also shown on the graph.

For an annulus with a larger size finite gap:

$$
\Omega_{c r}=\frac{41.19 \nu F_{g}}{r_{m}^{0.5}(b-a)^{1.5}}
$$

where $F_{g}$ is a geometrical factor defined by:

$$
F_{g}=\frac{\pi^{2}}{41.19 \sqrt{S}}\left(1-\frac{b-a}{2 r_{m}}\right)^{-1}
$$

and $S$ is given in an alternative form by:

$$
\begin{aligned}
S= & 0.0571\left(1-0.652 \frac{(b-a) / r_{m}}{1-(b-a) / 2 r_{m}}\right)+ \\
& 0.00056\left(1-0.652 \frac{(b-a) / r_{m}}{1-(b-a) / 2 r_{m}}\right)^{-1}
\end{aligned}
$$

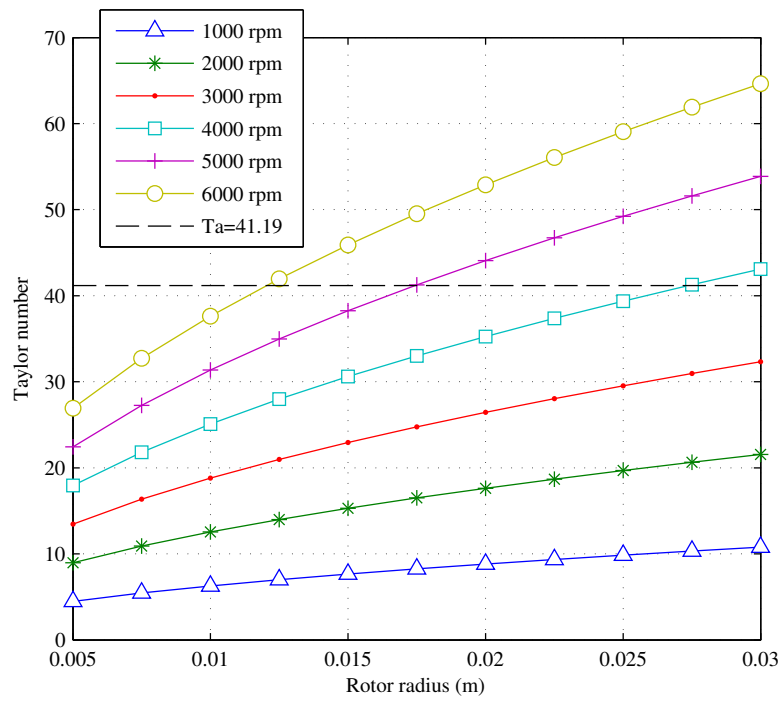

Fig. 2. Taylor number vs. rotor size \& speed for $0.5 \mathrm{~mm}$ gap machine

The wavelength for the instability is approximately $\lambda=$ $2(b-a)$.

\section{B. Convective heat transfer}

At low Taylor numbers the flow is a laminar shear flow and for $T a_{m}^{2} / F_{g}^{2}<1700$ heat transfer is dominated by conduction and can be predicted for inner or outer surface using the correlation developed by Bjorklund and Kays [14]:

$$
N u=\frac{h D_{h}}{k}=\frac{2[(b-a) / a]}{\ln [1+(b-a) / a]}
$$

where, for the case of an annulus, $D_{h}=2(b-a)$. For the machine geometry and speeds shown in figure 2 , this correlation would be applied when $T a<41.19$ and since the heat transfer in equation 19 is independent of speed and only a weak function of radius in this range, inserting values gives $h \approx 58 \mathrm{~W} / \mathrm{m}^{2} \mathrm{~K}$ for $5 \mathrm{~mm} \leq a \leq 30 \mathrm{~mm}$.

For laminar flow with vortices $\left(1700<T a_{m}^{2} / F_{g}^{2}<10^{4}\right)$, from Becker and Kaye [15], [16]:

$$
N u=0.128\left(T a^{2} / F_{g}^{2}\right)^{0.367}
$$

For the machine geometry and speeds shown in figure 2, this correlation would be applied when $T a>41.19$.

For turbulent flow $\left(10^{4}<T a_{m}^{2} / F_{g}^{2}<10^{7}\right)$, from Becker and Kaye [15], [16]:

$$
N u=0.409\left(T a^{2} / F_{g}^{2}\right)^{0.241}
$$

In equations 20 and 21 an annulus area based on the logarithmic mean radii $[(b-a) / \ln (a / b)]$, has been used to evaluate the overall heat transfer.

The preceding correlations all apply to the totally enclosed case with no superposed axial throughflow whereas the remainder of this section concerns the case of axial throughflow. Experiments using hot wire anemometry and flow visualisation 
by Kaye and Elgar [17] revealed the existence of four modes of flow in this situation. Their tests were performed using an annulus with inner and outer radii of $34.9 \mathrm{~mm}$ and 47.5 mm respectively at axial throughflow Reynolds numbers up to 2000 and rotational Reynolds numbers up to 3000. The four regimes of flow corresponded to:

1) Purely laminar flow

2) Laminar flow with Taylor vortices

3) Turbulent flow with vortices

4) Turbulent flow

For laminar flow with vortices, Simmers and Coney [18] used principles similar to the Reynolds analogy, which makes use of the fact that a similarity exists between the energy equation and momentum equation, for heat transfer between the outer annulus surface and throughflow, giving:

$$
N u=\frac{4 \operatorname{Pr} \operatorname{Re}_{z}^{0.5}\left(\Omega_{a}^{2} a b^{3} / \nu^{2}\right)^{0.3675}}{B(A /(1-x))^{0.5}(x /(1-x))^{0.25}\left(\Omega_{a}^{2} a b^{3} / \nu^{2}\right)_{c r}^{0.6175}}
$$

where:

$$
\begin{gathered}
A=\left[1+x^{2}+\left(\frac{1-x^{2}}{\ln x}\right)\right]\left(2+\frac{1-x^{2}}{\ln x}\right)^{-1} \\
B=\operatorname{Pr}+\ln \left\{1+\operatorname{Pr} \exp \left[\frac{2}{3}\left(\frac{1-x}{x}\right)^{0.25}\left(\frac{x A}{(1-x)^{2}}\right)^{0.5}\right.\right. \\
\left.\left.\operatorname{Re}_{z}^{-0.5}\left(\frac{\Omega_{a}^{2} a b^{3}}{\nu^{2}}\right)^{0.1325}\left(\frac{\Omega_{a}^{2} a b^{3}}{\nu^{2}}\right)_{c r}^{0.1175}-1\right]-\operatorname{Pr}\right\} \\
\operatorname{Re}_{z}=\frac{u_{z} D_{h}}{\nu}, \text { where } u_{z} \text { is axial velocity }
\end{gathered}
$$

The Prandtl number $\operatorname{Pr}$ is the ratio of viscous to thermal diffusion rates, defined as:

$$
\operatorname{Pr}=\frac{\mu c_{p}}{k}
$$

This is a fluid property; for gases $\operatorname{Pr} \approx 0.7$.

For turbulent flow with vortices, Gazley [19] gave the following correlation based on tests at axial and rotational Reynolds numbers of up to $1.2 \mathrm{e} 4$ and $1.1 \mathrm{e} 5$ respectively:

$$
N u=0.03 R e_{e}^{0.8}
$$

where the Reynolds number was based on the vector sum of the bulk axial flow and the rotor speed:

$$
R e_{e}=\frac{\sqrt{u_{z}^{2}+u_{\phi}^{2}} D_{h}}{\nu}
$$

Gazley looked at the effect of slotted features in the annulus, such as may be due to salient poles in an electrical machine, showing a corresponding small increase in heat transfer over the smooth surface condition.

Also for turbulent flow with vortices, Kosterin and Finatev [20], present the following correlation:

$$
N u=0.018\left\{R e_{z}\left[1+0.6\left(\left(\Omega_{a} a D_{h} / \nu\right) / R e_{z}\right)^{2}\right]^{0.5}\right\}^{0.8}
$$

for $0<\left(\Omega_{a} a D_{h} / \nu\right)<1 e 5$ and $3 e 3<R e_{z}<3 e 4$.

For turbulent flow in an annulus with inner cylinder rotation, Kuzay and Scott [21] present the following correlation based on measurements from a rig with a radius ratio of 0.57 for axial Reynolds numbers up to $6.5 \mathrm{e} 5$ and rotational Reynolds numbers up to $4 \mathrm{e} 4$ :

$$
N u=0.022\left[1+\left(\frac{D_{h} u_{\phi}}{\pi a u_{z}}\right)^{2}\right]^{0.8714} \operatorname{Re}_{z}^{0.8} \operatorname{Pr}^{0.5}
$$

Childs et al. [22] and Childs and Turner [23] present the following correlation based on experimental data for a large rotating drum of $400 \mathrm{~mm}$ diameter, for turbulent flow heat transfer in an annulus with stationary outer cylinder:

$$
\frac{N u-N u_{x}}{N u_{x}}=0.068\left(\frac{u_{\phi}}{u_{z}}\right)^{2}
$$

where $N u_{x}=0.023 R e_{z}^{0.8} \sqrt{P r}$, for $1.7 e 5<R e_{z}=<$ $3.7 e 5,1.4 e 4<R e_{\phi}=\frac{\Omega a^{2}}{\nu}<2.7 e 6$ and $1.65<z / D_{h}<5$.

\section{Worked example}

Consider a fully enclosed rotor $200 \mathrm{~mm}$ in diameter, running at $1500 \mathrm{rpm}$ in air with kinematic viscosity of $2 \mathrm{e}-5 \mathrm{~m}^{2} / \mathrm{s}$, with an annular gap of $1 \mathrm{~mm}$. Firstly, the critical speed is calculated from equation 15 , this evaluates to $\Omega_{c r}=82.2 \mathrm{rad} / \mathrm{s}$. Since the running speed of $1500 \mathrm{rpm}$ is greater than this $(157 \mathrm{rad} / \mathrm{s})$, it is likely that there are vortices in the annulus.

The heat transfer can now be calculated. First the quantity $T a^{2} / F_{g}^{2}$ is calculated using equations 8 and 17 . This equals to 6858. Since this is between 1700 and 10000, equation 20 may be used to calculate the Nusselt number, giving $N u=$ 3.27. From the definition of Nusselt number, the convective heat transfer coefficient is therefore equal to:

$$
h=\frac{N u k}{D_{h}}=47 \mathrm{~W} / \mathrm{m}^{2} \mathrm{~K}
$$

where the hydraulic diameter $D_{h}=2 \mathrm{~mm}$.

\section{DisC GEOMETRY MACHINES}

Disc geometry machines have their air-gap magnetic field oriented in the axial direction. The most common type of is the axial flux permanent magnet (AFPM) brushless machine, although other machines including brushed DC and induction machines are also possible. There are many different configurations, for example rotor-stator, rotor-stator-rotor, statorrotor-stator and multiple discs, and both totally enclosed and throughflow ventilated configurations are possible.

In a disc machine the air-gap flow is pumped by a pressure difference caused by the rotation of the rotor(s). This makes disc machines fundamentally different to cylindrical machines, where any axial throughflow of air must be pumped by an externally imposed pressure difference. Heat typically from 
the stator is transferred to the fluid by forced convection at the stator surface. The configuration of the airflow path is crucial to the thermal performance.

\section{A. Airflow in the rotor-stator gap}

A large body of analytical, numerical and experimental research has been undertaken concerning airflow and heat transfer of a disc system. This is relevant to electrical machines, gas turbines, turbochargers, brake discs and many other types of machine. Dorfman's book [24] concentrates on analytical solutions, with some experimental results. The book by Owen and Rogers [25] gives a comprehensive review of various analytical solutions and compares these with experimental data for free disc and rotor-stator systems with and without superposed flow, including heat transfer.

The simplest kind of rotating disc system is the 'free disc', an infinite radius rotating disc in a fluid. This was originally examined by von Kármán [26] who found a solution to the Navier-Stokes equations showing that the disc drags fluid from the rotor centre to the outside edge, at the same time drawing fresh fluid inwards axially as shown in figure 3. By assuming axi-symmetry, he reduced the partial differential equations (PDEs) to a set of four coupled ordinary differential equations (ODEs) and solved the non-dimensionalised equations for radial, tangential and axial velocity components and pressure as a function of axial distance, by using approximate analytical (momentum-integral) methods, for both laminar and turbulent flow.

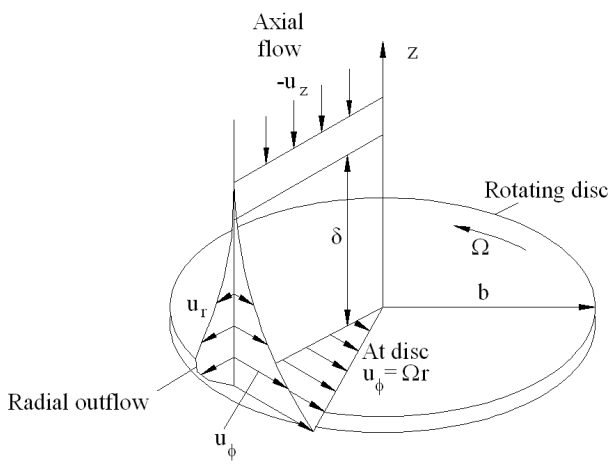

Fig. 3. Free rotor disc flow

The same approach can be applied to a rotating fluid above a stationary surface where it is found that the opposite effect occurs, i.e. the fluid near the surface is slowed down and moves radially inwards.

Batchelor [27] studied mathematically the flow between infinite coaxial discs using similar methods to von Kármán, proposing that particularly at large Reynolds numbers there would be an inviscid rotating fluid core between separate laminar or turbulent boundary layers located on the rotor and stator respectively. The rotation speed of the core is approximately $40 \%$ of the rotor disc speed. This type of rotorstator flow with core rotation is often called 'Batchelor flow'.
In the core itself the fluid rotates with almost zero radial and axial velocity and a radial pressure gradient balances the centrifugal force:

$$
\rho \frac{V_{\theta}^{2}}{r}=\frac{d p}{d r}
$$

Stewartson [28], alternatively to Batchelor, proposed that the tangential velocity drops away from the rotor surface to zero at the stator surface, with no core rotation. Until the 1980s there was some controversy over which solution was correct. However, both authors based their calculations on infinite diameter discs and subsequently it has been shown that with finite diameter discs, it is the condition at the boundary that determines whether the flow will tend toward a Batchelor or a Stewartson pattern. Batchelor flow is usually observed in enclosed systems, but in systems which are open at the periphery and have radial throughflow, Stewartson flow may be seen.

Soo [29] analysed laminar flow over an enclosed rotor using approximate methods and suggests that, for rotor cooling, radial outflow is more effective than radial inflow. Figure 4 shows a range of streamlines for the laminar case with open periphery and superposed in/out flow.

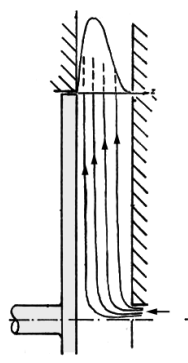

(a)

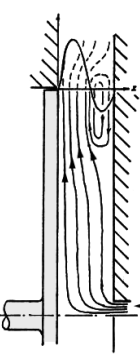

(b)

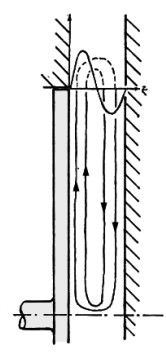

(c)

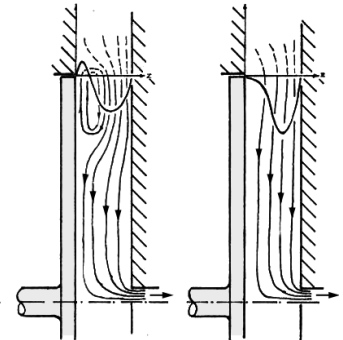

(d)

(e)
Fig. 4. Typical streamlines for laminar flow in small clearances with no case at the outer edge, from Soo [29]; (a) radial outflow, large pumping rate (b) radial outflow, smaller pumping rate (note separation bubble on stator) (c) no superposed flow (d) radial inflow, small pumping rate (e) radial inflow, large pumping rate

The smooth transition from Batchelor to Stewarston flow is illustrated by Poncet et al. [30], [31] who experimented with a rotor-stator system under three conditions: no superimposed throughflow, radial inflow, and radial outflow. They found that when a radial inflow is superimposed, the flow remains Batchelor type but when a radial outflow is superposed the flow becomes Stewartson type and the core rotation diminishes or disappears. The authors show that the transformation from Batchelor to Stewartson flow can be quantified directly.

For the case of an enclosed rotor-stator system with no throughflow, Daily and Nece [32] observed four distinct flow regimes depending on $G$ and $R e_{\theta}$, as follows:

1) Merged laminar rotor and stator boundary layers, occurring for small values of $G$ and $R e_{\theta}$.

2) Separate laminar boundary layers, at larger values of $G$ and small $R e_{\theta}$. 
3) Merged turbulent boundary layers at smaller $G$ values and larger $R e_{\theta}$.

4) Separate turbulent boundary layers at larger $G$ and $R e_{\theta}$ values.

Regimes 2 and 4 correspond to a Batchelor type flow with core rotation. The distinction between each regime is not sharp but occurs over a range of values of $G$ and $R e_{\theta}$.

In a disc type electrical machine a range of configurations are possible: the periphery may be fully enclosed, partially enclosed or open but surrounded by a grill or mesh. There may be significant rotor pumped radial throughflow. The flow may tend towards either a Batchelor or a Stewartson pattern depending on the particular configuration, and the actual flow pattern may be a combination of both regimes.

In throughflow ventilated disc machines, the size of the air inlet is very important in determining or limiting the amount of air flow through the system. Smaller air inlet sizes may restrict the air throughflow rate and therefore change the internal air gap flow regime. Rotating air inlets exhibit a variety of different discharge coefficients that depend on the rotational speed and incidence angle [33].

\section{B. Convective heat transfer}

A variety of techniques have been applied to measure heat transfer in rotor-stator systems. Cobb and Saunders [34] applying the Reynolds analogy found that the turbulent rotor heat transfer correlation for a free disc could be derived accurately from rotor torque measurements. Owen [35] also applied the Reynolds analogy to a turbulent rotor-stator system and extended the concept to include frictional heating effects.

A recent direct measurement technique involves numerical solution of the heat equation in a solid (either rotor or stator), using measured temperatures at various locations, for example applied by Owen, Bayley and Haynes [36] to measure both rotor and stator heat transfer. Infra-red (IR) thermography of rotor surface temperatures can be used with this method, as applied by Boutarfa et al. [37]. A thin layer of thermally insulating material (zirconia) is applied at the disc surface and the temperature at the aluminium-zirconia junction measured by embedded thermocouples. The heat flux through the rotor surface is found by solving the heat equation in the zirconia using a finite-difference method, applying the experimentally measured temperature boundary conditions. The surface heat flux is obtained from the temperature gradient at the surface.

A number of authors have derived and measured rotor heat transfer in the 'free disc' case (figure 3). The heat transfer correlations are usually given as a power law for average Nusselt number is a function of rotational Reynolds number:

$$
N u=A R e_{\theta}^{B}
$$

In the case of laminar flow $\left(R e_{\theta}<1 \mathrm{e} 5\right)$, Dorfman's analytical correlation [24] has $A=0.35$ and $B=0.5$; Cobb and Saunders [34] give a similar correlation for an isothermal free rotor, with $A=0.36$ and $B=0.5$. Finally, Owen and Rogers [25] derive the values $A=0.33$ and $B=0.5$ by numerical solution of the energy equation in the laminar free rotor case. Since in all of these cases $B=0.5$, on substituting the definitions of Nusselt number and rotational Reynolds number into equation 34, the radius $R$ cancels and a direct relationship between rotor speed and convective heat transfer coefficient may be found:

$$
h \approx 0.35 k\left(\frac{\Omega}{\nu}\right)^{0.5}
$$

A correlation for turbulent average Nusselt numbers (valid for $R e_{\theta}>2.8 e 5$ ) for an isothermal free rotor is given by Owen, Haynes and Bayley [36]:

$$
N u=0.0151 R e_{\theta}^{0.8}
$$

Boutarfa and Harmand [37] present experimentally measured correlations for rotor heat transfer in an open throughflow ventilated rotor-stator system with varying gap ratio $G$, with no additional superposed air flow. Their correlations are presented in table II with the four regimes corresponding to those given by Daily and Nece [32] previously. In all these cases the reference temperature for the convective heat transfer coefficient is the fluid inlet temperature, which is usually ambient. As can be seen at $G=0.01$, the rotor heat transfer is higher in the rotor-stator system compared with the free rotor, in both laminar and turbulent regimes. At intermediate gap sizes $0.02 \leq G \leq 0.06$ the authors found that the rotor heat transfer drops to a minimum at $G \approx 0.02$ which is lower than the free rotor value, then increases to the free rotor value at $G>0.06$.

TABLE II

ROTOR HEAT TRANSFER CORRELATIONS, BOUTARFA AND HARMAND [37]

\begin{tabular}{ccc}
\hline \hline$G$ & Regime & Correlation \\
\hline 0.01 & lam. & $N u=7.46 R e_{\theta}^{0.32}$ \\
$0.02-0.06$ & lam. & $N u=0.5\left(1+5.47 \times 10^{-4} e^{112 G}\right) R e_{\theta}^{0.5}$ \\
0.01 & turb. & $N u=0.044 R e_{\theta}^{0.75}$ \\
$0.02-0.06$ & turb. & $N u=0.033\left(12.57 e^{-33.18 G}\right) R e_{\theta}^{0.6+25 G^{12 / 7}}$ \\
\hline
\end{tabular}

In the literature, stator heat transfer has received less attention than rotor heat transfer, although it is important in the design of disc type machines since most of the heat is typically generated in the stator. Bunker et al. [38], with gas turbine internal air systems in mind, measured both rotor and stator heat transfer in a shrouded rotor-stator system with radial outflow using a central jet of air impinging on the rotor.

Recent work by Howey, Holmes and Pullen [39], [40] measured radially resolved stator convective heat transfer in a generalised throughflow ventilated disc machine, using a geometric mock-up of a machine. This comprised a heated stator surface adjacent to a $470 \mathrm{~mm}$ diameter spinning aluminium rotor driven by a servo motor, with air admitted through an opening at the stator centre and expelled at the periphery of the system. The gap size between rotor and stator was adjustable by inserting accurately machined spacers. Two 


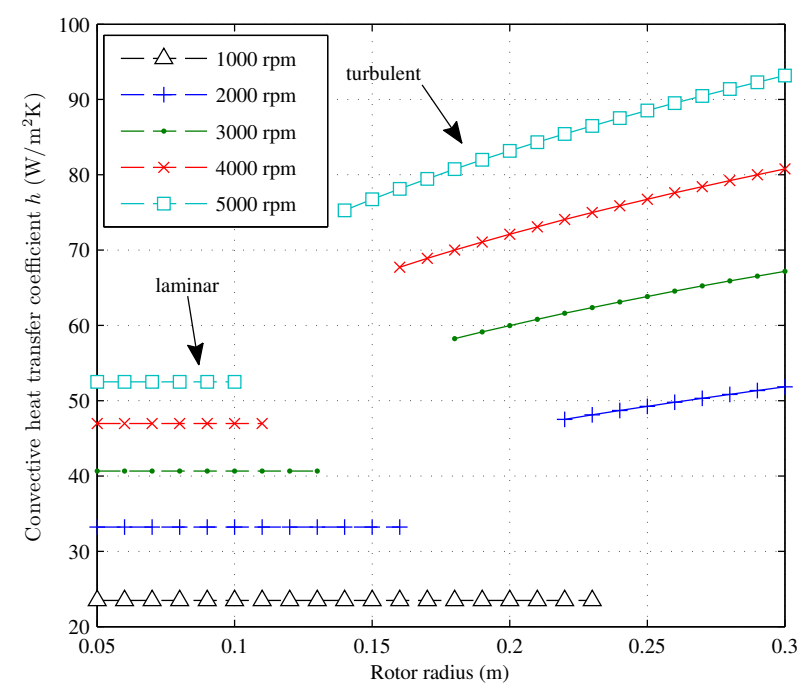

Fig. 5. Stator surface convective heat transfer coefficient, disc machine

types of rotor were tested: a flat rotor and also a rotor with protrusions designed to mimic the permanent magnets of an AFPM machine. Gap ratios $G$ from 0.01 to 0.05 were tested, with rotational Reynolds numbers $R e_{\theta}$ from $3.7 \mathrm{e} 4$ to 1e6. The measurements were undertaken using a thin-film heating technique, enabling direct measurement of heat flux by measurement of the electrical power into a heater array. It was found that average stator heat transfer results can be correlated according to the power law of equation 34, with constants $A$ and $B$ given in table III. These are valid in the turbulent regime, range $R e_{\theta} \geq 5.19 e 5$. All values of $B$ are similar, $B=0.673 \pm 0.028$ but values of $A$ differ according to gap ratio $G$. In the laminar regime $\left(R e_{\theta} \leq 3 e 5\right)$, it was found that the free rotor correlation can be reliably used at the stator to predict heat transfer.

TABLE III

AVERAGE STATOR HEAT TRANSFER TURBULENT CORRELATIONS

\begin{tabular}{ccc}
\hline \hline $\mathrm{G}$ & $\mathrm{A}$ & $\mathrm{B}$ \\
\hline 0.0106 & 0.0790 & 0.640 \\
0.0127 & 0.0888 & 0.633 \\
0.0170 & 0.0406 & 0.682 \\
0.0212 & 0.0315 & 0.691 \\
0.0297 & 0.0347 & 0.679 \\
0.0467 & 0.0234 & 0.712 \\
\hline
\end{tabular}

Figure 5 shows how the convective heat transfer coefficient at the stator surface varies with disc size and speed in the case of a disc type machine with gap ratio $G=0.01$, using equation 35 in the laminar case, and the data from the first row of table III in the turbulent case.

\section{Worked example}

Consider a disc type throughflow cooled machine with rotor $400 \mathrm{~mm}$ in diameter, running at $3000 \mathrm{rpm}$ in air with kinematic viscosity of $2 \mathrm{e}-5 \mathrm{~m}^{2} / \mathrm{s}$, with an axial rotor-stator gap of 4 $\mathrm{mm}$. Firstly the rotational Reynolds number is calculated from equation 3 based on the speed and rotor outside radius: $R e_{\theta}$ $=6.3 \mathrm{e} 5$ and it can be seen that the flow is turbulent both at the rotor and the stator surfaces. The gap ratio is $G=0.01$; therefore the appropriate rotor heat transfer correlation to use is $N u=0.044 R e_{\theta}^{0.75}$ from table II, and this evaluates to $\mathrm{Nu}$ $=982$ and therefore $h=126 \mathrm{~W} / \mathrm{m}^{2} \mathrm{~K}$.

The stator heat transfer can also be calculated using equation 34 with values for $A$ and $B$ from the first line of table III, since $G \approx 0.1$. This gives $N u_{s}=406$ and therefore $h_{s}=52$ $\mathrm{W} / \mathrm{m}^{2} \mathrm{~K}$ where in both cases the subscript $s$ refers to the stator.

\section{Conclusions}

Prediction of temperatures in electrical machines is vital to ensure that designs deliver the required performance at the lowest cost. This review paper has focused on air-gap convective heat transfer and has sought to give an overview of the work in this area, collecting and summarising the key correlations and findings, including worked examples which are particularly relevant to smaller machines. Many correlations have arisen from work on turbomachinery, but are equally applicable in electrical machine design and have not been presented previously in this context. It is hoped that designers can directly apply these correlations within thermal models to predict temperatures.

In all types of electrical machine, the air gap convective heat transfer is not constant and is affected primarily by the rotating speed of the machine and the size of the rotor and air gap. In general, larger and faster machines have higher convective heat transfer coefficients in the air gap, while in smaller slower machines the heat transfer coefficients are lower. Indeed smaller fractional horsepower machines may struggle to achieve very high torque densities compared to larger machines. This is because in a smaller machine the temperature gradients in the flow are lower and because the flow is more likely to be laminar. Introducing externally pumped superposed airflow will increase the heat transfer in the gap, as will increasing the surface roughness of internal flow passages.

Although this paper has been divided in to a section on cylindrical machines and a section on disc machines, both sections are relevant to the design of either type of machine since all machines have both 'disc' faces and a 'drum' section.

\section{REFERENCES}

[1] A. Boglietti, "Guest Editorial," IEEE Transactions on Industrial Electronics, vol. 55, no. 10, pp. 3498-3499, 2008.

[2] A. Boglietti, A. Cavagnino, D. Staton, M. Shanel, M. Mueller, and C. Mejuto, "Evolution and modern approaches for thermal analysis of electrical machines," IEEE Transactions on Industrial Electronics, vol. 56, no. 3, pp. 871-882, 2009.

[3] D. Staton, A. Boglietti, and A. Cavagnino, "Solving the more difficult aspects of electric motor thermal analysis in small and medium size industrial induction motors," IEEE Transactions on Energy Conversion, vol. 20, no. 3, pp. 620-628, 2005.

[4] A. Fitzgerald, C. Kingsley, and S. Umans, Electrical Machinery, 5th ed. McGraw-Hill Companies, 1990.

[5] J. Gieras, R. Wang, and M. Kamper, Axial flux permanent magnet brushless machines, 2nd ed. Springer Verlag, 2008. 
[6] C. Hernandez-Aramburo, T. Green, and S. Smith, "Assessment of power losses of an inverter-driven induction machine with its experimental validation," IEEE Transactions on Industry Applications, vol. 39, no. 4, pp. 994-1004, 2003.

[7] Engineering Sciences Data Unit, Flow in rotating components - discs, cylinders and cavities, ESDU data sheet 07004. IHS ESDU, 2007.

[8] P. Mellor, D. Roberts, and D. Turner, "Lumped parameter thermal model for electrical machines of TEFC design," Electric Power Applications, IEE Proceedings B, vol. 138, no. 5, pp. 205-218, 1991.

[9] E. Spooner and B. Chalmers, “TORUS': A slotless, toroidal-stator permanent-magnet generator," Electric Power Applications, IEE Proceedings $B$, vol. 139, no. 6, pp. 497-506, 1992.

[10] D. Staton and A. Cavagnino, "Convection Heat Transfer and Flow Calculations Suitable for Electric Machines Thermal Models," IEEE Transactions on Industrial Electronics, vol. 55, no. 10, pp. 3509-3516, 2008.

[11] P. Childs and C. Long, "A review of forced convective heat transfer in stationary and rotating annuli," Proceedings of the Institution of Mechanical Engineers Part C: Journal of Mechanical Engineering Science, vol. 210, pp. 123-134, 1996.

[12] L. Rayleigh, "On the dynamics of revolving fluids," Proceedings of the Royal Society, London, Series A, vol. 93, pp. 148-154, 1916.

[13] G. Taylor, "Stability of a viscous liquid contained between two rotating cylinders," Proceedings of the Royal Society, London, Series A, vol. 223, pp. 239-343, 1923.

[14] I. Bjorklund and W. Kays, "Heat transfer between concentric rotating cylinders," Journal of Heat Transfer, vol. 81, pp. 175-186, 1959.

[15] K. Becker and J. Kaye, "Measurements of diabatic flow in an annulus with an inner rotating cylinder," Trans. ASME, Journal of Heat Transfer vol. 84 , pp. $97-105,1962$.

[16] _ _ "The influence of a radial temperature gradient on the instability of fluid flow in an annulus with an inner cylinder rotating," Trans. ASME Journal of Heat Transfer, vol. 84, pp. 106-110, 1962.

[17] J. Kaye and E. Elgar, "Modes of adiabatic and diabatic flow in an annulus with inner rotating cylinder," Trans. ASME, vol. 80, pp. 753765,1958 .

[18] D. Simmers and J. Coney, "A Reynolds analogy solution for the heat transfer characteristics of combined Taylor vortex and axial flows," International Journal of Heat and Mass Transfer, vol. 22, pp. 679-689, 1979.

[19] C. Gazley, "Heat transfer characteristics of the rotational and axial flow between concentric cylinders," Trans. ASME, vol. 80, pp. 79-90, 1958

[20] S. Kosterin and Y. Finatev, "Heat transfer in turbulent airflow the annular space between rotating coaxial cylinders (in Russian)," Inzh. Fiz. Z. H., vol. 8, pp. 3-9, 1962.

[21] T. Kuzay and C. Scott, "Turbulent heat transfer studies in annulus with inner cylinder rotation," ASME Journal of Heat Transfer, vol. 99, pp 12-19, 1977.

[22] P. Childs, A. Turner, C. Vaughan, D. Rayner, and F. Bayley, "Heat transfer to a rotating drum in an annulus with a stator blade row and axial throughflow," ASME Paper 92-GT-249, 1992.

[23] P. Childs and A. Turner, "Heat transfer on the surface on the surface of a cylinder rotating in an annulus at high axial and rotational Reynolds number," Proc. 10th International Heat Transfer Conference, Brighton, $U K$, vol. 3, pp. 13-18, 1994.

[24] L. Dorfman, Hydrodynamic Resistance and the Heat Loss of Rotating Solids. Oliver \& Boyd, 1963.

[25] J. Owen and R. Rogers, Flow and Heat Transfer in Rotating-Disc Systems, Vol. 1: Rotor-Stator Systems, 1st ed. Research Studies Press, 1989.

[26] T. von Kármán, "Über laminare und turbulente Reibung," Z. Angew. Math. Mech, vol. 1, no. 4, pp. 233-235, 1921.

[27] G. Batchelor, "Note on a class of solutions of the Navier-Stokes equations representing steady rotationally-symmetric flow," The Quarterly Journal of Mechanics and Applied Mathematics, vol. 4, no. 1, pp. 29$41,1951$.

[28] K. Stewartson, "On the flow between two rotating coaxial discs," Mathematical Proceedings of the Cambridge Philosophical Society, vol. 49, no. 2, pp. 333-341, 1953.

[29] S. Soo, "Laminar flow over an enclosed rotating disk," Transactions of the ASME, vol. 80, no. 2, pp. 287-296, 1958.

[30] S. Poncet, M. Chauve, and R. Schiestel, "Batchelor versus Stewartson flow structures in a rotor-stator cavity with throughflow," Physics of Fluids, vol. 17, p. 075110,2005

[31] S. Poncet, R. Schiestel, and M. Chauve, "Centrifugal flow in a rotorstator cavity," ASME Journal of Fluids Engineering, vol. 127, p. 787, 2005 .
[32] J. Daily and R. Nece, "Chamber dimension effects on induced flow and frictional resistance of enclosed rotating disks," ASME J. Basic Eng, vol. 82, no. 1, pp. 217-232, 1960.

[33] A. Idris, K. Pullen, and D. Barnes, "An investigation into the flow within inclined rotating orifices and the influence of incidence angle on the discharge coefficient," Proceedings of the Institution of Mechanical Engineers, Part A: Journal of Power and Energy, vol. 218, no. 1, pp. 55-68, 2004

[34] E. Cobb and O. Saunders, "Heat transfer from a rotating disk," Proceedings of the Royal Society of London. Series A, Mathematical and Physical Sciences, vol. 236, no. 1206, pp. 343-351, 1956.

[35] J. Owen, "The Reynolds analogy applied to flow between a rotating and a stationary disc," International Journal of Heat and Mass Transfer, vol. 14 , pp. 451-460, 1971.

[36] J. Owen, C. Haynes, and F. Bayley, "Heat Transfer from an Air-Cooled Rotating Disk," Proceedings of the Royal Society of London. Series A, Mathematical and Physical Sciences, vol. 336, no. 1607, pp. 453-473, 1974.

[37] R. Boutarfa and S. Harmand, "Local convective heat transfer for laminar and turbulent flow in a rotor-stator system," Experiments in Fluids, vol. 38, no. 2, pp. 209-221, 2005.

[38] R. Bunker, D. Metzger, and S. Wittig, "Local Heat Transfer in Turbine Disk Cavities: Part I, Rotor and Stator Cooling With Hub Injection of Coolant," ASME Journal of Turbomachinery, vol. 114, p. 211, 1992.

[39] D. Howey, A. Holmes, and K. Pullen, "Radially resolved measurement of stator heat transfer in a rotor-stator disc system," International Journal of Heat and Mass Transfer, vol. 53, no. 1-3, pp. 493-501, 2010.

[40] - "Prediction and measurement of heat transfer in air-cooled disctype electrical machines," Proceedings of the IET, Power Electronics, Machines and Drives (PEMD) conference, 19-21 April 2010, Brighton UK, 2010.

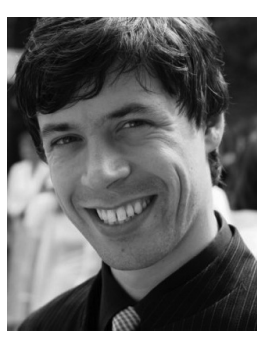

David A. Howey is a Research Associate in the Mechanical Engineering Department at Imperial College London. He received the M.Eng degree in Electrical and Information Sciences from Cambridge University (2002) and the Ph.D from Imperial Col lege (2010), investigating air gap convective heat transfer in disc type electrical machines. His current research interests include: thermal analysis of electrical machines and batteries; cm-scale wind turbines for energy harvesting and electrically assisted turbochargers for large off-road diesel engine vehicles.

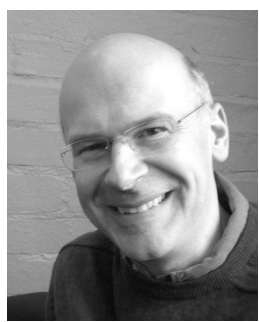

Peter R.N. Childs is the Professorial Lead in Engineering Design at Imperial College London where he leads the Design Engineering group and is joint course director for the Innovation Design Engineering degree at the Royal College of Art and Imperial. His general interests include: creativity and the use of creativity tools; mechanical design rotating flow; heat transfer and sustainable energy. $\mathrm{He}$ was formerly the director of InQbate, a Centre of Excellence in Teaching and Learning in Creativity and director of the Rolls-Royce supported University Technology Centre for Aero-Thermal Systems at the University of Sussex. He has recently published a book on rotating flow.

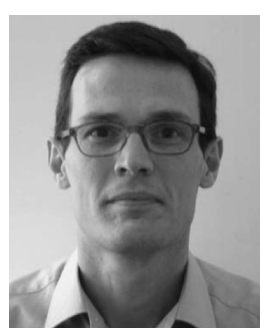

Andrew S. Holmes received the B.A. degree in natural sciences from Cambridge University (1987) and the $\mathrm{Ph} . \mathrm{D}$. degree in electrical engineering from Imperial College London (1992). He is currently Professor of Microelectromechanical Systems with the Optical and Semiconductor Devices Group, Department of Electrical and Electronic Engineering, Imperial College London. His research interests are mainly in the areas of micropower generation and conversion, MEMS devices for microwave applications, and laser processing for MEMS manufacture. 